\title{
ON THE CONVOLUTION EQUATION $P=P * Q$ OF CHOQUET AND DENY FOR PROBABILITY MEASURES ON SEMIGROUPS
}

\author{
A. MUKHERJEA
}

ABSTRACT. In this paper, the problem of determining regular probability measures $P$ and $Q$ satisfying $P=P * Q=Q * P$ has been solved on locally compact cancellative semigroups. The results also hold in the complete metric case. The results have been used to determine the primitive idempotent measures on locally compact semigroups.

1. We consider the representation of a regular (relative to compact sets from inside) Borel (generated by open sets) probability measure $P$ as a convolution product of itself and some other regular Borel probability measure $Q$ :

$$
P=P * Q
$$

where the convolution $P * Q$ is defined to be the unique regular probability measure such that for every $f \in C(S)=$ the continuous functions with compact support on $S$, a locally compact Hausdorff semigroup,

$$
\int f(x) P * Q(d x)=\iint f(x y) P(d x) Q(d y) .
$$

By a result in $[\mathbf{H}-\mathbf{R}]$ (see also [M-T]), it follows that the function $x \rightarrow$ $P\left(B x^{-1}\right)$ is measurable (even when $S$ is only completely regular Hausdorff) for every Borel set $B$ where

$$
B x^{-1}=\{y \in S: y x \in B\} \text { and } P * Q(B)=\int P\left(B x^{-1}\right) Q(d x) .
$$

When $S$ is only completely regular Hausdorff, by $P * Q$, we will mean the measure defined by (2). The solutions of (1) have been exhibited for probability as well as infinite measures in the case of a locally compact abelian topological group by Choquet and Deny in [C-D] and later by Tortrat for probability measures on topological groups in [T]. Tortrat

Received by the editors April 29, 1971.

AMS 1969 subject classifications. Primary 2875, 6008.

Key words and phrases. Locally compact topological semigroup, regular measure, convolution of measures, normed Haar measure.

(c) American Mathematical Society 1972 
proved the following theorem: When $S$ is a topological group, $Q$ is a solution of (1) if and only if $P(B)=P\left(B x^{-1}\right)$ for every $x$ in the smallest closed group containing the support of $Q$ and every Borel set $B$ in $S$.

The purpose of our paper is to investigate solutions of $P=P * Q=Q * P$ for probability measures $P$ and $Q$ on locally compact or metric topological (Hausdorff) semigroups. The infinite case has been dealt with in [M]. Here are our results:

THEOREM 1. Let $S$ be a locally compact Hausdorff (or metric) bicancellative semigroup. Then $Q$ is a solution of $P=P * Q=Q * P$ if and only if $P\left(B x^{-1}\right)=P\left(x^{-1} B\right)=P(B)$ for every Borel set $B$ and $x$ in the smallest closed semigroup $H$ generated by $F^{\prime}$, the support of $Q$. If $Q$ is a solution and $F$ (the support of $P$ ) is contained in $H$, then $P$ is a normed Haar measure on $F$ which becomes a compact group, provided $S$ is complete in the case when $S$ is metric. Hence a regular probability measure is idempotent on $S$ if and only if it is a normed Haar measure on a compact topological group, which is its support.

THEOREM 2. Let $S$ be a locally compact Hausdorff (or complete metric) semigroup. Let $P$ and $Q$ be two idempotent probability measures with supports $F$ and $F^{\prime}$ respectively such that $P=P * Q=Q * P$. Then $P=Q$ if and only if $F \cap F^{\prime} \neq \varnothing$ and $F^{\prime}$ contains a subgroup maximal in $F \cup F^{\prime}$.

Other results on primitive idempotent measures are given in Theorem 3 and as remarks following Theorem 3 .

The theorems will be proved in $\$ 3$. The necessary lemmas will be given in $\S 2$. [Note that in Theorem 2 above, by an idempotent measure $P$, we meant $P^{2}=P * P=P$.]

2. From now on, $F$ and $F^{\prime}$ will denote always the supports of the regular probability measures $P$ and $Q$ respectively. When $S$ is locally compact, Glicksberg in [G] has shown that for every separately continuous bounded function $f$ on $S \times S$,

$$
\iint f(x, y) P(d x) Q(d y)=\iint f(x, y) Q(d y) P(d x)
$$

so that it follows that

$$
P * Q(B)=\int P\left(B x^{-1}\right) Q(d x)=\int Q\left(x^{-1} B\right) P(d x)
$$

from [H-R]. When $S$ is metric, $F$ and $F^{\prime}$ are both separable (being closures of sigma-compact sets) so that using Fubini's theorem on the separable metric space $F \times F^{\prime}$ for the product measure $P \times Q$, it is easy to see that (3) is valid. 
In this section, $S$ is always a locally compact or metric topological (Hausdorff) semigroup. Our main lemma (Lemma 3) is based upon an idea used in [M-T]. The first two lemmas are well known and stated without proof. $F$ and $F^{\prime}$ are supports of $P$ and $Q$ respectively.

LEMMA 1. F is the smallest closed set with P-measure 1.

Note that $F=\{x \in S: P(V)>0$ for every open $V, x \in V\}$.

LEMMA 2. If $U$ is any open set and $x_{\beta}$ is a net converging to $x$, then $P\left(U x^{-1}\right) \leqq \lim \inf P\left(U x_{\beta}^{-1}\right)$.

Lemma 3. Let $P=P * Q=Q * P$. Then for every Borel set $B$ and $x \in F$, $y \in F^{\prime}$, we have

$$
P\left(B x^{-1}\right)=P\left(B x^{-1} y^{-1}\right) \text { and } P\left(x^{-1} B\right)=P\left(y^{-1} x^{-1} B\right) .
$$

Proof. We will only prove the first equality since the second one will follow similarly because of (3).

First we observe that for every bounded measurable function $f(s)$ on $S$, we have

$$
\int f(s) P(d s)=\iint f(s t) P(d s) Q(d t)=\iint f(s t) Q(d s) P(d t)
$$

since $P=P * Q=Q * P$. [Here the double integrals are in iterated sense.]

Let $K$ be any compact set and $x \in F$. Let $P\left(K x^{-1}\right)=a$. Let $\varepsilon$ be an arbitrary positive number. Then by the regularity of $P_{x}$ (where $P_{x}(B)=$ $P\left(B x^{-1}\right)$ ), we can find open sets $W$ and $O$ and a closed set $V$ such that $O \supset V \supset W \supset K$ and $P\left(O x^{-1}\right)<a+\varepsilon$ Let $A=\left\{s: P\left(V s^{-1}\right) \geqq a+\varepsilon\right\}$. Then the complement of $A, A^{c}$ is open by Lemma 2. Since $x \in A^{c} \cap F, P\left(A^{c}\right)>0$. Let $g(s)=\max \left\{P\left(V s^{-1}\right)-a-\varepsilon, 0\right\}$. Then by the remark in the previous paragraph, we have

$$
\int\left[\int g(s t) Q(d s)-g(t)\right] P(d t)=0 .
$$

Since $g(t) \leqq \int g(s t) Q(d s)$, it follows that for some Borel set $E$ with $P(E)=0$ and for all $t$ in $S-E$, we have $g(t)=\int g(s t) Q(d s)$ Let $y \in A^{c}-E$. Then $g(y)=0$ and therefore, $g(s y)=0$ for $Q$-almost all $s$. Since $W$ is open and $W \subset V$, by Lemma $2, P\left(W y^{-1} s^{-1}\right) \leqq a+\varepsilon$ for all $s$ in $F^{\prime}$. If $U$ is any open set containing $x$, then $U \cap A^{c} \cap F$, being nonempty and relatively open in $F$, has positive $P$-measure and hence it must intersect $A^{c}-E$. Using Lemma 2 on $P_{s}$, we have then $P\left(W x^{-1} s^{-1}\right) \leqq a+\varepsilon$ for every $s$ in $F^{\prime}$. Since $K \subset W$, $P\left(K x^{-1} s^{-1}\right) \leqq P\left(K x^{-1}\right)+\varepsilon$ for every $s$ in $F^{\prime}$. Since $\varepsilon$ is arbitrary and $P\left(K x^{-1}\right)=\int P\left(K x^{-1} s^{-1}\right) Q(d s)$, we have $P\left(K x^{-1}\right)=P\left(K x^{-1} s^{-1}\right)$ for $Q$-almost all $s$ and hence for all $s$ in $F^{\prime}$, by Lemma 2. The lemma follows now from the regularity of $P$. Q.E.D. 
Lemma 4. Let $S, P$ and $Q$ be as in Lemma 3. Then if there exists $x$ in $F$ such that $y x=z x \Rightarrow y=z$, then for every Borel set $B, P(B)=P\left(B s^{-1}\right)$ for every $s$ in the smallest closed semigroup $H$ generated by $F^{\prime}$.

Proof. Since right cancellativity by $x$ is permitted, $K x x^{-1}=K$ for every compact set $K$. If $s \in F^{\prime}$, by Lemma $3, P(K)=P\left((K x) x^{-1}\right)=$ $P\left((K x) x^{-1} s^{-1}\right)=P\left(K s^{-1}\right)$. Since $P$ is regular, $P(B)=P\left(B s^{-1}\right)$ for every $s$ in $F^{\prime}$ and for all Borel sets $B$. This equality is trivially true then for every $s$ in the semigroup generated by $F^{\prime}$. Using Lemma 2 and regularity of $P$, the equality follows for all $s$ in $H$. Q.E.D.

We quote the following lemma (proved in [M-T]) without proof.

LeMma 5. Let $S$ be a locally compact Hausdorff or complete metric topological semigroup. Let $P(B)=P\left(B x^{-1}\right)=P\left(x^{-1} B\right)$ for all $x$ in $S$ and all Borel sets $B$. Then $F$ is a compact topological group which is an ideal of $S$.

Before we go into $\S 3$, we need the following definition. A semigroup $S$ is called completely simple if it is simple and contains a primitive idempotent. The following facts are well known (see p. 61 in [B-H]). Let $S$ be completely simple and $E(S)=$ the idempotents in $S$. Then

(1) for $e \in E(S), e S e$ is a maximal subgroup $(=G)$,

(2) $X=E(S e)$ is a left-zero semigroup and $Y=E(e S)$ is a right-zero semigroup and $S$ is topologically isomorphic to $X \times G \times Y$ (with product topology, $S$ being taken as locally compact), where the multiplication is given by $(x, g, y)\left(x^{\prime}, g^{\prime}, y^{\prime}\right)=\left(x, g y x^{\prime} g^{\prime}, y^{\prime}\right)$.

3. We are now ready to prove Theorem 1 and Theorem 2 .

Proof of Theorem 1. First we observe that, in Lemma 4 if $S$ was left cancellative by $x$, then $P(B)=P\left(s^{-1} B\right)$ for every $s$ in $H$. Then Theorem 1 is immediate from Lemma 4 and Lemma 5. Q.E.D.

Proof of Theorem 2. By [M-T], we know that $F$ and $F^{\prime}$ are both completely simple. Since $\operatorname{cl}\left(F F^{\prime}\right)=\operatorname{cl}\left(F^{\prime} F\right)=F$ and $F \cap F^{\prime} \neq \varnothing,\left(F \cap F^{\prime}\right) F^{\prime} \subset$ $F \cap F^{\prime}$ and $F^{\prime}\left(F \cap F^{\prime}\right) \subset F \cap F^{\prime}$ so that $F \cap F^{\prime}$ is an ideal of $F^{\prime}$. This means that $F^{\prime} \subset F$. If $F^{\prime}$ contains a maximal subgroup, then it must be $e F^{\prime} e$, for some idempotent $e$ in $F^{\prime}$. Now $F$ can be identified with the semigroup $E(F e) \times e F e \times E(e F)$ and $F^{\prime}$ with the subsemigroup $E\left(F^{\prime} e\right) \times e F^{\prime} e \times E\left(e F^{\prime}\right)$. Since $\operatorname{cl}\left(F F^{\prime}\right)=\operatorname{cl}\left(F^{\prime} F\right)=F$, it follows that $E(F e)=E\left(F^{\prime} e\right)$ and $E(e F)=$ $E\left(e F^{\prime}\right)$. Also $e F^{\prime} e=e F e$ since $e F^{\prime} e$ is maximal. This means that $F=F^{\prime}$. Now since $P=P * Q=Q * P$, using Lemma 3 , we have for every $x$ in $F$,

$$
P\left(B x^{-1}\right)=\int Q\left(B x^{-1} y^{-1}\right) P(d y)=\int Q\left(B x^{-1}\right) P(d y)=Q\left(B x^{-1}\right)
$$


and

$$
P(B)=\int P\left(B x^{-1}\right) Q(d x)=\int Q\left(B x^{-1}\right) Q(d x)=Q(B),
$$

for every Borel set $B$. Q.E.D.

REMARK. In a locally compact semigroup, it can so happen that $P=P * Q=Q * P, P$ and $Q$ both idempotent probability measures, but $P$ is neither a zero of $P(S)=$ the regular probability measures on $S$ (algebraically a semigroup under convolution) nor $P=Q$. This is shown by the following example. Let $S_{1}$ and $S_{2}$ be any two locally compact completely simple semigroups supporting respectively the idempotent probability measures $P$ and $Q$. [We can always find such $S_{1}$ and $S_{2}$, see [P] or [M-T].] Let us also assume that $S_{1} \cap S_{2}=\varnothing$ and $S_{1}$ is not a group. Let $S=S_{1} \cup S_{2}$. Let the topology of $S$ be the union of the topologies of $S_{1}$ and $S_{2}$. We define in $S$ multiplication by $x y=y x=x$ for every $x$ in $S_{1}$ and $y$ in $S_{2}$ so that $S_{1}$ and $S_{2}$ are both subsemigroups of $S$. Then $S$ becomes a locally compact topological semigroup. One can now easily verify that $P=$ $P * Q=Q * P$, considering natural extensions of $P$ and $Q$ on $S$. But $P \neq Q$.

An idempotent probability measure $Q$ is called primitive if $P=P * Q=$ $Q * P, P$ idempotent probability $\Rightarrow P=Q$. These measures have been studied by Collins in $[\mathbf{C}]$ on compact groups and by Pym in $[\mathbf{P}]$ on compact semigroups. [In the above, it is assumed that $P$ is not a zero of $P(S)$.] From Lemma 5, we see that if $P(S)$ has a zero, then $S$ has a kernel which is a group; and if in addition to $P(S)$ having a zero, $S$ is right (or left) cancellative, then $S$ itself is a topological group which is compact, in which case the primitive idempotents are all known in $P(S)$ from the work of Collins in [C]. In the case when $S$ does not happen to be a group, we have the following theorem.

THEOREM 3. Let $S$ be a locally compact right (or left) cancellative Hausdorff semigroup which is not a group. Then an idempotent probability measure is primitive if and only if its suppo't contains a maximal compact subgroup.

Proof. Let $Q^{2}=Q, P^{2}=P, P$ and $Q$ in $P(S)$ and $P=P * Q=Q * P$. By [M-T], $F$ and $F^{\prime}$ are both completely simple. Also since $S$ is right cancellative and $F$ is an ideal of $F \cup F^{\prime}, F^{\prime} \subset F$; for, if $x \in F^{\prime}$ and $e \in E(F)$, then $x e=y$ is in $F$ so that $x e=y e$ which means $x \in F$. Hence by Theorem 2, $P=Q$ if $F^{\prime}$ contains a maximal subgroup.

Conversely, let $Q$ be a primitive idempotent in $P(S)$. Then by Lemma 4, $Q\left(B x^{-1}\right)=Q(B)$ for every Borel set $B$ and $x \in F^{\prime}$. By Theorem 4.9, p. 94 in [B-H], since $F^{\prime}$ is a left group now, we can find a $Q_{1}$ in $P(S)$ with its support $E\left(F^{\prime}\right)$ and a normed Haar measure $Q_{2}$ in $P(S)$ with its support 
$e F^{\prime}$ where $e$ is a fixed idempotent in $F^{\prime}$ such that $Q=Q_{1} \times Q_{2}$. It then follows that $Q=Q_{1} * Q_{2}$. Suppose that $F^{\prime}$ does not contain a maximal compact subgroup. Since $e F^{\prime}$ is a compact group (being the support of a finite Haar measure), there is a compact group $G$ properly containing $e F^{\prime}$. Let $Q_{3}$ be the normed Haar measure of $G$. Let $P=Q_{1} * Q_{3}$. Then $P \neq Q$. If $\beta_{e}$ is the point mass at $e$, then $\beta_{e} * Q_{1}$ has support contained in $G$ and since $Q_{3}$ is a zero of $P(G)$ and $Q_{3}=Q_{3} * \beta_{e}$, it follows easily that $P=P * Q=Q * P$. This gives a contradiction that $Q$ is primitive. Q.E.D.

REMARK. Because of the example in the previous remark, we see that the cancellation condition cannot be removed from the theorem above. Also it may be relevant to mention that if $Q^{2}=Q$ and $Q \in P(S)$, where $S$ is a locally compact or complete metric semigroup and $Q * \beta_{x}=\beta_{x} * Q$ for every point mass $\beta_{x}$ for $x \in F^{\prime}$, then $F^{\prime}$ is a compact group and $Q$ is the normed Haar measure of $F^{\prime}$; since, by [M-T], we know that $F^{\prime}$ is a completely simple semigroup (closed) and also since for $x \in F^{\prime}$ then $\operatorname{cl}\left(F^{\prime} x\right)=F^{\prime} x$ and $\operatorname{cl}\left(x F^{\prime}\right)=x F^{\prime}$, we have $F^{\prime} x=x F^{\prime}$ for $x \in F^{\prime}$ which means that $F^{\prime} x=x F^{\prime}=F^{\prime}$ since $F^{\prime} x=x F^{\prime}$ is an ideal of $F^{\prime}$ which is simple. Note that when $S$ is metric, $F^{\prime}$ is separable and the well-known result of Montgomery that a complete metric separable topological semigroup which is algebraically a group is a topological group applies and then by the converse of Haar's theorem (see Theorem 1 in [0]), $F^{\prime}$ becomes compact.

On a locally compact semigroup, an idempotent probability measure $P$ can be expressed as $P_{1} * P_{2} * P_{3}$ where $P_{1}$ and $P_{3}$ are in $P(S)$ with supports respectively $E(F e)$ and $E(e F), e$ being some fixed idempotent in $F$ and $P_{2}$ is the normed Haar measure on the compact group $e F e$. [To see this, we notice that $P=\left(P * \beta_{e}\right) *\left(\beta_{e} * P\right), \beta_{e}$ being the point mass at $e$ and $P * \beta_{e}(B)=$ $P * \beta_{e}\left(B x^{-1}\right), \beta_{e} * P(B)=\beta_{e} * P\left(x^{-1} B\right)$ for every $x$ in $F$ by Lemma 3 so that as in the proof of Theorem 3, using Theorem 4.9, p. 94 in [B-H], we can write $P * \beta_{e}=P_{1} * P_{2}$ and $\beta_{e} * P=P_{2} * P_{3}$.] Now the following result can be obtained following the proof of the 'converse' part of Theorem 3: On a locally compact Hausdorff semigroup $S$, the support of a nonzero primitive idempotent probability measure must contain a compact subgroup maximal and proper in $S$ and be completely simple.

\section{REFERENCES}

[B-H] J. F. Berglund and K. H. Hofmann, Compact semitopological semigroups and weakly almost periodic functions, Lecture Notes in Math., no. 42, Springer-Verlag, Berlin and New York, 1967. MR 36 \#6531.

[C] H. S. Collins, Primitive idempotents in the semigroup of measures, Duke Math. J. 27 (1960), 397-400. MR 22 \#5691.

[C-D] G. Choquet and J. Deny, Sur l'équation de convolution $\mu=\mu * \sigma$, C.R. Acad. Sci. Paris 250 (1960), 799-801. MR 22 \#9808. 
[G] I. Glicksberg, Weak compactness and separate continuity, Pacific J. Math. 11 (1961), 205-214. MR 22 \#11275.

[H-R] M. Heble and M. Rosenblatt, Idempotent measures on a compact topological semigroup, Proc. Amer. Math. Soc. 14 (1963), 177-184. MR 30 \#214.

[M] A. Mukherjea, The convolution equation $P=P * Q$ of Choquet and Deny and relatively invariant measures on semigroups (the infinite case), Ann. Inst. Fourier (Grenoble) 21 (1971).

[M-T] A. Mukherjea and N. Tserpes, Idempotent measures on locally compact semigroups, Proc. Amer. Math. Soc. 29 (1971), 143-150.

[O] J. C. Oxtoby, Invariant measures in groups which are not locally compact, Trans. Amer. Math. Soc. 60 (1946), 215-237. MR 8, 253.

[P] J. S. Pym, Idempotent measures on semigroups, Pacific J. Math. 12 (1962), 685698. MR 26 \#6298.

[T] A. Tortrat, Lois de probabilité sur un espace topologique complètement régulier et produits infinis à termes indépendants dans un groupe topologique, Ann. Inst. Henri Poincaré Sect. B 1 (1964/65), 217-237. MR 31 \#2755.

Department of Mathematics, University of South Florida, Tampa, Florida 33620 